\title{
Randomized, Double-Blind, Placebo-Controlled Trial Demonstrates the Efficacy and Safety of Oral Aripiprazole for the Treatment of Tourette's Disorder in Children and Adolescents
}

\author{
Floyd Sallee, MD, Eva Kohegyi, MD, Joan Zhao, PhD, Robert McQuade, PhD, \\ Kevin Cox, MD, Raymond Sanchez, MD, ${ }^{2}$ Alet van Beek, BSc, ${ }^{3, *}$ Margaretta Nyilas, MD, \\ William Carson, $\mathrm{MD}^{2}$, and Roger Kurlan, $\mathrm{MD}^{4}$
}

\begin{abstract}
Objectives: Aripiprazole modulates dopaminergic and serotonergic pathways that may play a role in the pathogenesis of Tourette's disorder (TD). This trial evaluated the efficacy and safety of oral aripiprazole in the suppression of tics in children and adolescents with TD.

Methods: This phase 3, randomized, double-blind, placebo-controlled trial (ClinicalTrials.gov, NCT01727700) recruited patients who were 7-17 years old with a diagnosis of TD from hospitals, private practices, and research clinics at 76 sites in the United States, Canada, Hungary, and Italy. Patients were randomized in a 1:1:1 ratio by using an interactive voice/ web-response system to low-dose aripiprazole ( $5 \mathrm{mg} /$ day if $<50 \mathrm{~kg} ; 10 \mathrm{mg}$ /day if $\geq 50 \mathrm{~kg}$ ), high-dose aripiprazole $(10 \mathrm{mg} /$ day if $<50 \mathrm{~kg} ; 20 \mathrm{mg} /$ day if $\geq 50 \mathrm{~kg}$ ), or placebo for 8 weeks. Randomization was stratified by region (North America or Europe) and baseline body weight $(<50 \mathrm{~kg}$ vs. $\geq 50 \mathrm{~kg}$ ). The primary efficacy endpoint was mean change from baseline to week 8 in the Yale Global Tic Severity Scale Total Tic Score (YGTSS-TTS) for the intent-to-treat population.

Results: Between November 2012 and May 2013, 133 patients were recruited and randomized to low-dose aripiprazole $(n=44)$, high-dose aripiprazole $(n=45)$, or placebo $(n=44)$. Least-squares mean treatment differences versus placebo in change from baseline to week 8 in the YGTSS-TTS were statistically significant (high dose, -9.9 [95\% confidence interval, CI, -13.8 to -5.9$]$, low dose, -6.3 [95\% CI, -10.2 to -2.3$])$. At week 8, 69\% (29/42) of patients in the low-dose and 74\% (26/ 35) of patients in the high-dose aripiprazole groups demonstrated a Clinical Global Impression-Tourette's Syndrome improvement score of 1 (very much improved) or 2 (much improved) compared with 38\% (16/42) in the placebo group. The most common adverse events (AEs) were sedation (low dose, 8/44 [18.2\%], high dose, $4 / 45$ [8.9\%], placebo, 1/44 [2.3\%]), somnolence (low dose, $5 / 44$ [11.4\%], high dose, $7 / 45$ [15.6\%], placebo, 1/44 [2.3\%]), and fatigue (low dose, $3 / 44$ [6.8\%], high dose, $7 / 45$ [15.6\%], placebo, 0). No serious AEs or deaths occurred.
\end{abstract}

Conclusions: This study indicates that oral aripiprazole is a safe and effective treatment for tics in children and adolescents with TD.

Keywords: aripiprazole, Tourette's disorder, tics, adolescent, children

Previous presentation: Once-Daily Oral Aripiprazole for Treatment of Tics in Children and Adolescents With Tourette's Disorder: A Randomized, Double-Blind, Placebo-Controlled Trial. Presented at the American College of Neuropsychopharmacology Annual Meeting; Phoenix, AZ; December 7-14, 2014.

${ }^{1}$ Department of Psychiatry, University of Cincinnati College of Medicine, Cincinnati, Ohio.

${ }^{2}$ Otsuka Pharmaceutical Development \& Commercialization, Inc., Princeton, New Jersey.

${ }^{3}$ Mylan, Canonsburg, Pennsylvania.

${ }^{4}$ Atlantic Neuroscience Institute, Overlook Medical Center, Summit, New Jersey.

*Employed by Otsuka at the time this research was conducted.

Funding: Study design, data collection, analysis, and interpretation of results were funded by Otsuka Pharmaceutical Development \& Commercialization, Inc.

(C) Floyd Sallee et al. 2017; Published by Mary Ann Liebert, Inc. This is an Open Access article distributed under the terms of the Creative Commons Attribution License, which permits unrestricted use, distribution, and reproduction in any medium, provided the original work is properly cited. 


\section{Introduction}

$\mathbf{T}$ OURETTE'S DISORDER (TD) IS a neuropsychiatric condition that is characterized by the appearance of tics — sudden, rapid, and recurrent motor movements and/or vocalizations (Bitsko et al. 2014). The most severe tics occur between 6 and 15 years of age and can negatively affect quality of life (e.g., social and academic functioning) (Leckman et al. 1998; Cutler et al. 2009). For $>30$ years, haloperidol and pimozide were the only U.S. Food and Drug Administration (FDA)-approved pharmacotherapies for TD (US Food and Drug Administration 1984; Haloperidol tablet 2011) that led to off-label use of atypical antipsychotics along with behavior therapies (e.g., habit reversal therapy), for symptom relief (Dutta and Cavanna 2013). The Orphan Drug Act of 1983 (US Food and Drug Administration 2013), with origins from advocates for TD, was created to stimulate the development of new, verified treatments for rare diseases such as TD. Aripiprazole received orphan designation from the FDA for "treatment of Tourette's Syndrome."

Although the exact pathogenesis of TD is unknown, atypical antipsychotics became a part of the treatment paradigm because dopaminergic and serotonergic disturbances were implicated (Steeves and Fox 2008). The atypical antipsychotic aripiprazole is a dopamine $\mathrm{D}_{2^{-}}$and serotonin 5-hydroxytryptamine $(5-\mathrm{HT})_{1 \mathrm{~A}}$ receptor partial agonist and $5-\mathrm{HT}_{2 \mathrm{~A}}$ receptor antagonist (Abilify ${ }^{\circledR}$ 2016) that is approved by the FDA for the treatment of TD. Efficacy was demonstrated in a placebo-controlled clinical study that enrolled patients who were 6-18 years of age (Yoo et al. 2013). Aripiprazole is also indicated for children and adolescents with schizophrenia (ages 13-17), bipolar I disorder (ages 10-17), and irritability associated with autistic disorder (ages 6-17) (Abilify ${ }^{\circledR}$ 2016).

In previous small (e.g., $n<100)$ or uncontrolled studies in patients with TD, treatment with oral aripiprazole demonstrated efficacy in the suppression of tics while being generally well tolerated (Yoo et al. 2011, 2013; Rizzo et al. 2012; Wenzel et al. 2012). Here, we present results from the largest multinational, double-blind study to date that evaluated the efficacy and safety of oral aripiprazole in children and adolescents with TD and was part of the clinical evidence for the FDA approval of aripiprazole.

\section{Methods}

\section{Study design}

This was a phase 3, multicenter, randomized, double-blind, placebo-controlled trial that included patients from hospitals, private practices, and research clinics at 76 sites in the United States, Canada, Hungary, and Italy (NCT01727700; list of investigators who screened or enrolled patients available in "Supplementary Data; Supplementary Data are available online at www.liebertpub.com/cap). Study protocol, amendments, informed consent forms, and patient recruitment materials were reviewed and approved by institutional review boards or independent ethics committees for each investigational site and/or country. This study was conducted in compliance with the International Conference on Harmonisation Good Clinical Practice and applicable local laws and regulatory requirements.

\section{Patients}

Eligible patients were aged 7-17 years with a diagnosis of TD (Diagnostic and Statistical Manual of Mental Disorders, Fourth Edition, Text Revision [DSM-IV-TR]) (American Psychiatric Association 2000), confirmed by the Kiddie Schedule for Affective Disorders and Schizophrenia-Present and Lifetime Version, including the Diagnostic Supplement 5 (Substance Abuse and Other
Diseases, i.e., Tic Disorders); with a Yale Global Tic Severity Scale Total Tic Score (YGTSS-TTS) $\geq 20$ at screening and baseline (randomization); and with tic symptoms causing impairment in normal routines. Patients with a history of psychotic disorders and those with primary mood disorders (DSM-IV-TR), other neurologic disorders that may have accompanying abnormal movements, autism spectrum disorder, or severe obsessive-compulsive disorder were excluded. Also excluded were patients taking psychostimulants for attention-deficit/hyperactivity disorder who experienced new or exacerbated treatment-related tics and those with a psychoactive-substance use disorder within the previous three months (DSM-IV-TR) and/or a positive drug screen. A full list of inclusion and exclusion criteria is available in Supplementary Table S1. Patients who were poor metabolizers of cytochrome P450 (CYP) 2D6 were not included in the study.

All patients' guardians/legal representatives provided written informed consent, and all patients provided written informed assent at screening by using a written informed consent form and an informed assent form, respectively. The forms were based on local regulatory requirements and the International Conference on Harmonisation Good Clinical Practice Guideline and adhered to ethical principles of the Declaration of Helsinki.

\section{Randomization and masking}

Study investigators enrolled eligible patients between November 1, 2012 and May 20, 2013, and patients were randomly assigned via an interactive voice-response system/interactive web response in a 1:1:1 ratio to low-dose aripiprazole $(<50 \mathrm{~kg}$ at baseline, $5 \mathrm{mg} /$ day; $\geq 50 \mathrm{~kg}$ at baseline, $10 \mathrm{mg} /$ day), high-dose aripiprazole $(<50 \mathrm{~kg}$ at baseline, $10 \mathrm{mg} /$ day; $\geq 50 \mathrm{~kg}$ at baseline, $20 \mathrm{mg} /$ day), or placebo for 8 weeks.

Randomization codes used a fixed-block, computer-generated randomization schedule stratified by region (North America vs. Europe) and baseline body weight ( $<50 \mathrm{~kg}$ vs. $\geq 50 \mathrm{~kg}$ ). The study sponsor's biostatistics department provided the random allocation schedule that was designed to assign patients in a 1:1:1 ratio to a treatment regimen by using a block size of three. Blinding was achieved by using placebo tablets that were identical in appearance to the aripiprazole tablets. Treatment assignment codes were available only to an independent biostatistician. Except in cases of emergency unblinding, patients, investigational site personnel, study sponsors, and all other trial personnel remained blinded to treatment assignments.

The study included a pretreatment phase for a maximum of 42 days in length with screening and washout (where applicable), and a treatment phase with baseline visit on day 0 . On treatment phase completion, patients could enter an open-label study; those who discontinued the trial or who did not enter into the open-label extension were followed for $30 \pm 3$ days (Fig. 1).

Treatment with aripiprazole was initiated at $2 \mathrm{mg} /$ day and then increased to $5 \mathrm{mg} /$ day after 2 days of treatment. Thereafter, patients $<50 \mathrm{~kg}$ who were randomized to low-dose aripiprazole remained on $5 \mathrm{mg} /$ day, whereas patients $\geq 50 \mathrm{~kg}$ and patients in the high-dose groups had their daily doses increased stepwise by $5 \mathrm{mg} /$ day at the end of each week of treatment until they reached their target randomized dose (Supplementary Fig. S1). The target dose for patients $<50 \mathrm{~kg}$ was $5 \mathrm{mg} /$ day (low dose) or $10 \mathrm{mg} /$ day (high dose). The target dose for patients $\geq 50 \mathrm{~kg}$ was $10 \mathrm{mg} /$ day (low dose) and $20 \mathrm{mg} /$ day (high dose). If the randomized dose was not tolerated, a one-time dose reduction to the next lower dose level or to $2 \mathrm{mg} /$ day for the 5-mg/day group was permitted. 


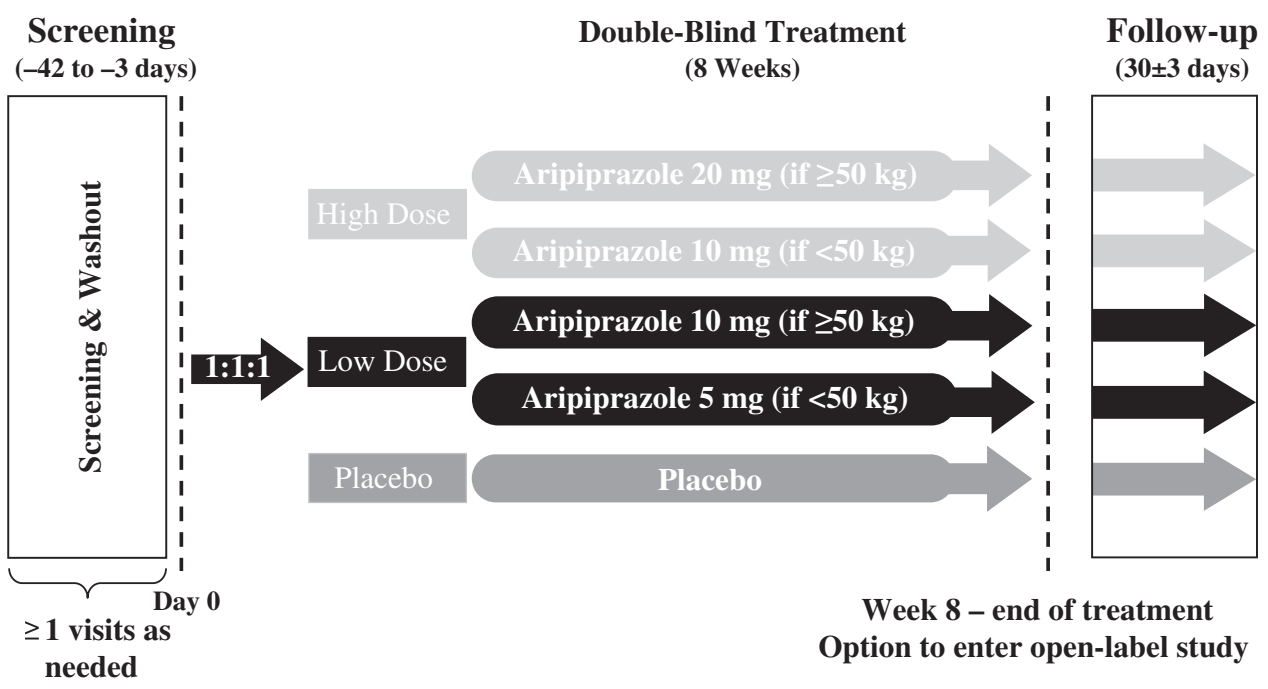

FIG. 1. Study design.

Clinic visits were scheduled at the start of weeks 1, 2 ( \pm 1 day), 4, 6 , and 8 ( \pm 3 days), when efficacy, safety, and other outcomes were assessed. Telephone calls to parents or guardians of patients were made at the start of weeks 3,5 , and 7 ( \pm 1 day) to confirm safety and tolerability.

\section{Outcomes}

The primary efficacy endpoint was the YGTSS-TTS mean change from baseline to week 8 . The YGTSS is a semi-structured scale that measures tic severity within the previous week. The YGTSS-TTS is a summation of motor and vocal tic severity scores (scale: 0-5) across five dimensions: number, frequency, intensity, complexity, and interference (Leckman et al. 1989). The YGTSS also includes an impairment rating scale that focuses on psychosocial aspects (Leckman et al. 1989). Subgroup analyses for the primary efficacy endpoint were based on age (7-12 years vs. 13-17 years), baseline YGTSS-TTS ( $<30$ vs. $\geq 30$ ), and region (North America or Europe). All raters were trained on the YGTSS by ProPhase, LLC (New York, NY) in face-to-face training. A rater certificate was granted on meeting inter-rater reliability standards. Raters were re-assessed every 6 months after they received their initial certificate.

The key secondary efficacy endpoint was the week 8 Clinical Global Impression-Tourette's Syndrome (CGI-TS) improvement scale score. Additional efficacy outcomes included a mean change from baseline to week 8 in total YGTSS score and CGI-TS severity score, response rates ( $>25 \%$ improvement from baseline to endpoint in YGTSS-TTS or a CGI-TS improvement score of 1 [very much improved] or 2 [much improved]), and treatment discontinuation rates. A more stringent post hoc analysis of response, defined as percentage of patients with $>50 \%$ improvement from baseline on YGTSS-TTS, was also performed. Additional post hoc efficacy outcomes included change from baseline in YGTSS total motor tic score, total vocal tic score, and impairment score.

Other outcomes (described briefly in Supplementary Table S2) included Swanson, Nolan, and Pelham-IV rating scale (SNAP-IV; assesses inattention, hyperactivity/impulsivity, and oppositional defiant symptoms), Children's Yale-Brown Obsessive Compulsive Scale (CY-BOCS), Children's Depression Rating Scale-Revised (CDRS-R), and Pediatric Anxiety Rating Scale (PARS) scores.
Safety and tolerability endpoints included treatment-emergent adverse events (AEs), vital signs, electrocardiograms, body weight, and clinical laboratory tests. The mean change in weight from baseline at the end-of-treatment visit was calculated, and a potentially clinically relevant weight change was defined as $\geq 7 \%$ increase or decrease from baseline. The Simpson-Angus Scale (SAS), the Abnormal Involuntary Movement Scale (AIMS), and the Barnes Akathisia Rating Scale (BARS) assessed extrapyramidal symptoms (Supplementary Table S2). The Columbia-Suicide Severity Rating Scale (C-SSRS) evaluated suicidality (Supplementary Table S2).

\section{Statistical analyses}

Sample size was estimated at 126 patients to provide $\geq 80 \%$ power to detect a treatment difference (standard deviation, SD) of -5 (8.5) between placebo and at least one out of two aripiprazole doses in the primary endpoint. Efficacy was analyzed in all randomized patients (intent-to-treat [ITT] population) and in randomized patients with no major protocol violations (per-protocol population). Prespecified major protocol violations included $<80 \%$ compliance with the investigational medicine product and use of prohibited medication that had a potential effect on efficacy evaluation.

The primary efficacy and key secondary endpoints, including subgroup and subscale analyses, were analyzed by using a mixedmodel repeated-measures (MMRM) linear model (time variable: visit week) with statistical significance at alpha level 0.05 (twosided) and treatment, region, weight group, and visit week as factors; baseline YGTSS-TTS as a covariate (for the primary efficacy endpoint); and treatment-by-week interactions in the model. The Hochberg procedure was used to adjust for multiplicity in testing two comparisons (low- or high-dose aripiprazole vs. placebo) of the primary and key secondary endpoints. If both doses demonstrated statistically significant improvements compared with placebo for the primary endpoint, then the key secondary endpoint was tested with comparisons for both doses versus placebo. Adjustments were not made for multiple comparisons over time (i.e., by treatment week).

Changes from baseline to week 8 in total YGTSS and CGI-TS severity scores were summarized by using descriptive statistics and compared between aripiprazole groups and placebo by fitting an 
MMRM linear model to the change scores (reported as least squares [LS] means). The repeated-measures generalized linear model was used to analyze the ordinal responses of CGI Improvement and the change from baseline in CGI Severity, respectively. Fixed categorical effects included treatment, week, treatment-by-week interaction, region, weight group, and baseline CGI severity score, and week was the time variable for repeated measures.

Response rates, including the post hoc analysis of proportion of patients with $>50 \%$ improvement from baseline in YGTSS-TTS, were analyzed by using observed case data, and treatment discontinuation rates were analyzed by using all randomized patients; both adjusted for region and weight group by using the CochranMantel-Haenszel method. No multiplicity adjustments were applied to any of the other efficacy endpoints (i.e., $p$-values unadjusted for multiplicity were reported). Pre-specified (a priori) statistical analyses were performed by using SAS version 9.3; SAS version 9.4 was used for post hoc analyses.

Patients in the ITT population who received $\geq 1$ dose of study medication were analyzed for safety (safety population). C-SSRS scores and AEs were analyzed by using descriptive statistics. SNAP-IV, CY-BOCS, CDRS-R, PARS, SAS, AIMS, and BARS scores were also analyzed by using an MMRM linear model.

\section{Results}

Of the 171 patients screened between November 2012 and May 2013, 133 were randomized at 76 sites in four countries: the United States $(n=92)$, Canada $(n=27)$, Hungary $(n=9)$, and Italy $(n=5)$. Overall, 119 patients (89.5\%) completed the study (Fig. 2). Demographics and baseline clinical characteristics were similar across treatment groups; however, the high-dose aripiprazole group had fewer black and more Asian patients, and the low-dose aripiprazole group had a lower mean body weight versus the other treatment groups. The majority of patients were men, white, weighed $<50 \mathrm{~kg}$, and ranged in age from 7 to 17 years (Table 1). The use of medi- cation for any reason before the start of study medication was reported in $26(59.1 \%)$ and $31(68.9 \%)$ patients in the low- and high-dose aripiprazole groups, respectively, and in $22(50.0 \%)$ patients in the placebo group.

Before the start of study medication, psychostimulants constituted the most frequent class of medication used overall by patients for any reason $(n=43[32.3 \%])$, whereas the use of anxiolytics and antipsychotics to treat TD was reported in 13 (29.5\%), 12 $(26.7 \%)$, and $8(18.2 \%)$ patients in the aripiprazole low-dose, high-dose, and placebo groups, respectively. The most common (i.e., $>5 \%$ in any treatment group) antipsychotics used to treat TD before the start of study medication were risperidone (low dose, $n=8$ [18.2\%], high dose, $n=5[11.1 \%]$, placebo, $n=4[9.1 \%])$, haloperidol (low dose, $n=3[6.8 \%]$, high dose, $n=3[6.7 \%]$, placebo, $n=2[4.5 \%]$ ), and aripiprazole (low dose, $n=3$ [6.8\%], high dose, $n=2$ [4.4\%], placebo, $n=2$ [4.5\%]). Prior use of anxiolytics to treat TD was low (i.e., $<2.5 \%$ ) across treatment groups.

A statistically significant improvement was demonstrated for the change from baseline to week 8 in YGTSS-TTS in the low-dose $(p=0.002)$ and high-dose $(p<0.0001)$ groups versus placebo (ITT population; Table 2) and remained significant after adjustment for multiple testing. Statistically significant differences versus placebo were observed at each time point from weeks 1 to 8 for high-dose aripiprazole and at all but week 2 for low-dose aripiprazole (Fig. 3). A noticeable separation occurred between the low- and high-dose groups from weeks 4 to 8 .

To demonstrate that improvements with aripiprazole were observed independent of the baseline disease severity or age, subgroup analyses for the primary efficacy endpoint were conducted; improvements versus placebo were demonstrated in patients with baseline YGTSS-TTS severity $<30$ (low dose: $p=0.0119$, high dose: $p=0.0005$ ) or baseline YGTSS-TTS severity $\geq 30$ (low dose: $p=0.0421$, high dose: $p=0.0029$ ), and in patients $7-12$ years (low dose: $p=0.0157$, high dose: $p=0.0002$ ) or 13-17 years (low dose: $p=0.0551$, high dose: $p=0.0204)$. Similarly, subgroup analysis to

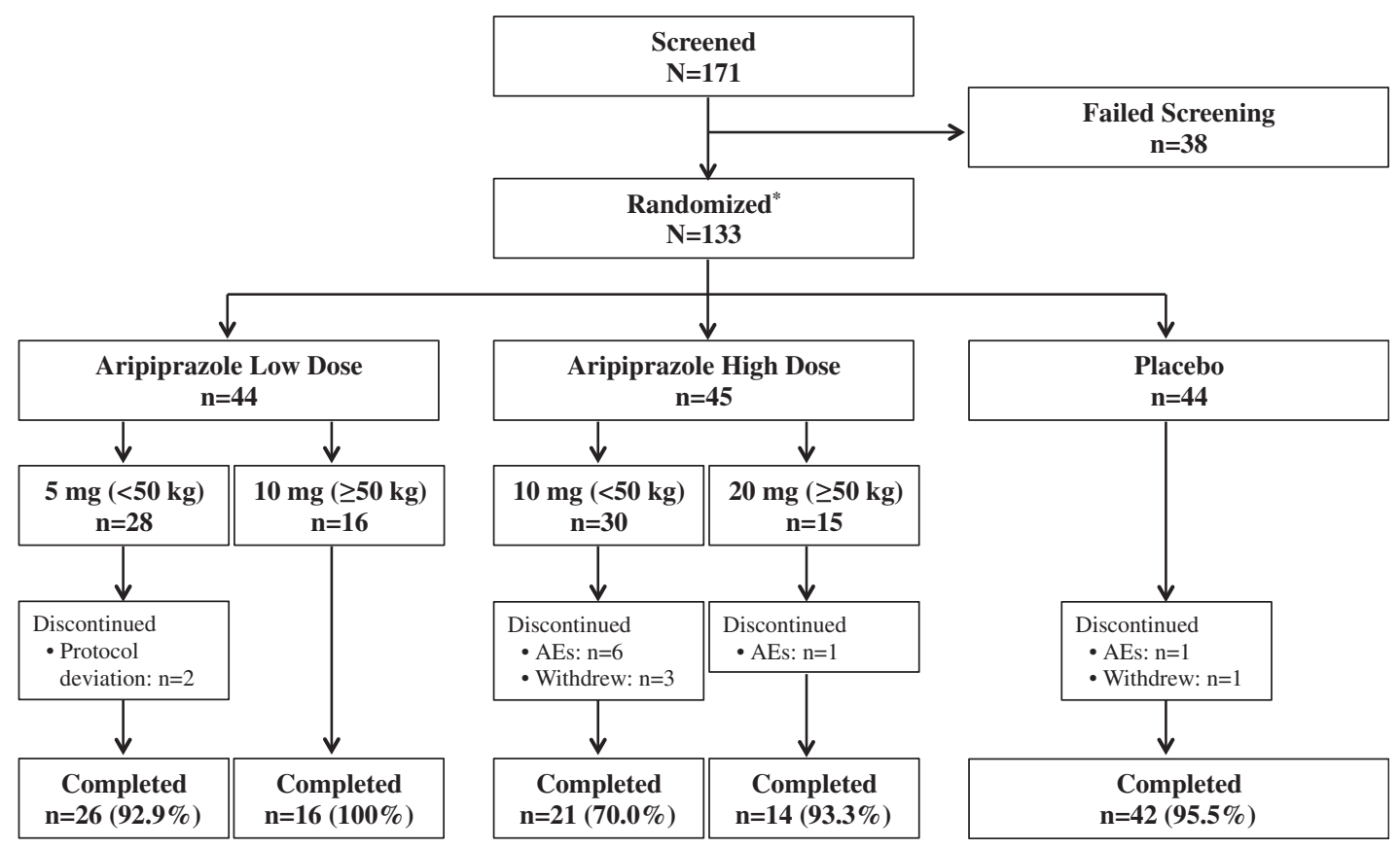

FIG. 2. Patient disposition. Reasons for screen failure were not specified. *Intent-to-treat sample and safety sample. AEs, adverse events. 
Table 1. Demographics and Baseline Characteristics (Intent-to-Treat Population)

\begin{tabular}{|c|c|c|c|}
\hline & \multicolumn{2}{|c|}{ Aripiprazole } & \multirow[b]{2}{*}{ Placebo $(\mathrm{n}=44)$} \\
\hline & Low dose $(\mathrm{n}=44)$ & High dose $(\mathrm{n}=45)$ & \\
\hline Age, mean (SD) [range], years & $11.1(3.1)[7.0-17.0]$ & $11.8(2.8)[7.0-17.0]$ & $11.6(2.8)[7.0-17.0]$ \\
\hline Men, $n(\%)$ & $36(81.8)$ & $35(77.8)$ & $33(75.0)$ \\
\hline \multicolumn{4}{|l|}{ Race, $n(\%)^{\mathrm{a}}$} \\
\hline White & $38(86.4)$ & $39(86.7)$ & $39(88.6)$ \\
\hline Black & $6(13.6)$ & $1(2.2)$ & $4(9.1)$ \\
\hline Asian & 0 & $3(6.7)$ & 0 \\
\hline Native American or Alaskan Native & 0 & $1(2.2)$ & 0 \\
\hline Other & 0 & $1(2.2)$ & $1(2.3)$ \\
\hline Weight, mean (SD), kg & $44.2(16.0)$ & $47.4(20.1)$ & $47.8(21.8)$ \\
\hline \multicolumn{4}{|l|}{ Weight group, $n(\%)^{\mathrm{a}}$} \\
\hline$<50 \mathrm{~kg}$ & $28(63.6)$ & $30(66.7)$ & $29(65.9)$ \\
\hline$\geq 50 \mathrm{~kg}$ & $16(36.4)$ & $15(33.3)$ & $15(34.1)$ \\
\hline Total Tic Severity Score, mean (SD) & $29.2(5.6)$ & $31.2(6.4)$ & $30.7(6.0)$ \\
\hline CGI-TS severity score, mean (SD) & $4.3(0.6)$ & $4.1(1.1)$ & $4.2(0.9)$ \\
\hline Normal to borderline ill (score $1-2), n(\%)^{\mathrm{b}}$ & $0(0.0)$ & $5(11.1)$ & $2(4.5)$ \\
\hline Mildly to moderately ill (score $3-4), n(\%)^{\mathrm{b}}$ & $27(61.4)$ & $25(55.6)$ & $27(61.4)$ \\
\hline Markedly to severely ill (score $5-6), n(\%)^{\mathrm{b}}$ & $17(38.6)$ & $15(33.3)$ & $14(31.8)$ \\
\hline Total YGTSS Score, mean (SD) & $61.2(11.3)$ & $62.5(13.5)$ & $62.8(12.1)$ \\
\hline CY-BOCS Total Score, mean (SD) & $2.3(4.5)$ & $3.3(5.6)$ & $2.8(4.4)$ \\
\hline PARS Total Anxiety Score, mean (SD) & $3.4(4.0)$ & $4.9(5.4)$ & $3.5(3.9)$ \\
\hline CDRS-R Score, mean (SD) & $23.3(7.4)$ & $22.4(4.5)$ & $21.6(4.8)$ \\
\hline \multicolumn{4}{|l|}{ SNAP-IV Subscale Scores, mean (SD) } \\
\hline Inattention & $1.3(0.8)$ & $1.4(0.8)$ & $1.4(0.8)$ \\
\hline Hyperactivity/impulsivity & $1.0(0.8)$ & $1.1(0.8)$ & $1.1(0.8)$ \\
\hline $\mathrm{ADD} / \mathrm{ADHD}$ & $1.2(0.7)$ & $1.3(0.7)$ & $1.3(0.7)$ \\
\hline
\end{tabular}

Total YGTSS score ranges from 0 to 100, and CGI-TS score ranges from 1 to 7 . Higher scores represent more severe symptoms, and a greater reduction from baseline represents a greater improvement. SNAP-IV subscale scores range from 0 to 3, CY-BOCS total score ranges from 0 to 40 , obsessions total and compulsions total scores range from 0 to 25 , PARS total score ranges from 0 to 35 , and CDRS-R total score ranges from 17 to 113 . Higher scores represent a worse condition, and a larger reduction from baseline represents greater improvement.

${ }^{\mathrm{a} B}$ Based on number of patients randomized.

${ }^{\mathrm{b}}$ One patient in the placebo group did not have baseline CGI-TS severity score.

ADD/ADHD, attention-deficit disorder/attention-deficit/hyperactivity disorder; CDRS-R, Children's Depression Rating Scale-Revised; CGI-TS, Clinical Global Impression-Tourette's Syndrome; CY-BOCS, Children's Yale-Brown Obsessive Compulsive Scale; ITT, intent-to-treat; PARS, Pediatric Anxiety Rating Scale; SD, standard deviation; SNAP-IV, Swanson, Nolan, and Pelham-IV rating scale; YGTSS, Yale Global Tic Severity Scale.

rule out regional differences showed improvements versus placebo in patients from North America (low dose: $p=0.0129$, high dose: $p<0.0001$ ) and Europe (low dose: $p=0.0456$, high dose: $p=0.0714)$. In the high-dose aripiprazole group, significant improvements from baseline at week 8 were demonstrated on the YGTSS motor tic, vocal tic, and impairment scores $(p<0.0001$, $p=0.0001$, and $p=0.0001$, respectively; Table 2). In the low-dose aripiprazole group, patients demonstrated significant improvements from baseline at week 8 on the YGTSS motor tic and impairment scores ( $p=0.0002$ and $p=0.0033$ ), and improvement on the vocal tic score approached statistical significance $(p=0.0563$; Table 2).

Significant improvement in the CGI-TS improvement score at week 8 was demonstrated in both aripiprazole groups $(p=0.0010$ and $p=0.0002$ for low- and high-dose groups, respectively) versus placebo (Table 2); treatment differences remained significant after adjustment for multiple testing. Differences versus placebo for both aripiprazole groups were statistically significant at each time point from weeks 1 to 8 (Supplementary Fig. S3); the treatment effect of both aripiprazole groups was comparable at each time point. When CGI-TS improvement scores were analyzed as ordinal data, the odds of having a score lower than the placebo group (indicating a greater degree of improvement) were significant with low-dose
$(\mathrm{OR}=6.2 ; p<0.0001)$ and high-dose $(\mathrm{OR}=6.3 ; p<0.0001)$ aripiprazole at week 8 (also see Supplementary Fig. S2). Although CGI-TS improvement scores did not differ between low- and highdose aripiprazole groups $(\mathrm{OR}=0.98 ; p<0.9612)$, treatment effect sizes were numerically larger in the high-dose versus low-dose group for the total YGTSS-TTS and YGTSS-TTS total motor tic, total vocal tic, and impairment scores.

Differences in total YGTSS score and CGI-TS severity scores for both aripiprazole groups versus placebo were significant at week 8 (Table 2) and at each time point, with the exception of week 2 in the low-dose group (Supplementary Figs. S4 and S5). When analyzed as ordinal data, the odds of having a greater improvement in CGI-TS severity score were approximately six times higher for high-dose aripiprazole than for the placebo group $(\mathrm{OR}=6.1$, $p<0.001)$ and approximately five times higher for low-dose aripiprazole than for the placebo group ( $\mathrm{OR}=4.9, p=0.001)$.

A consistent and significantly greater response rate versus placebo was observed in both dose groups for all study weeks except week 8 in the low-dose group (Table 2 and Supplementary Fig. S6). A response was observed as early as week 1 in both aripiprazole groups and increased throughout the trial in all treatment groups. At week 8, the response rate in the low-dose aripiprazole group was not significantly different from placebo $(p=0.0835$, 
Table 2. EfFicacy Endpoints (Intent-to-Treat Population)

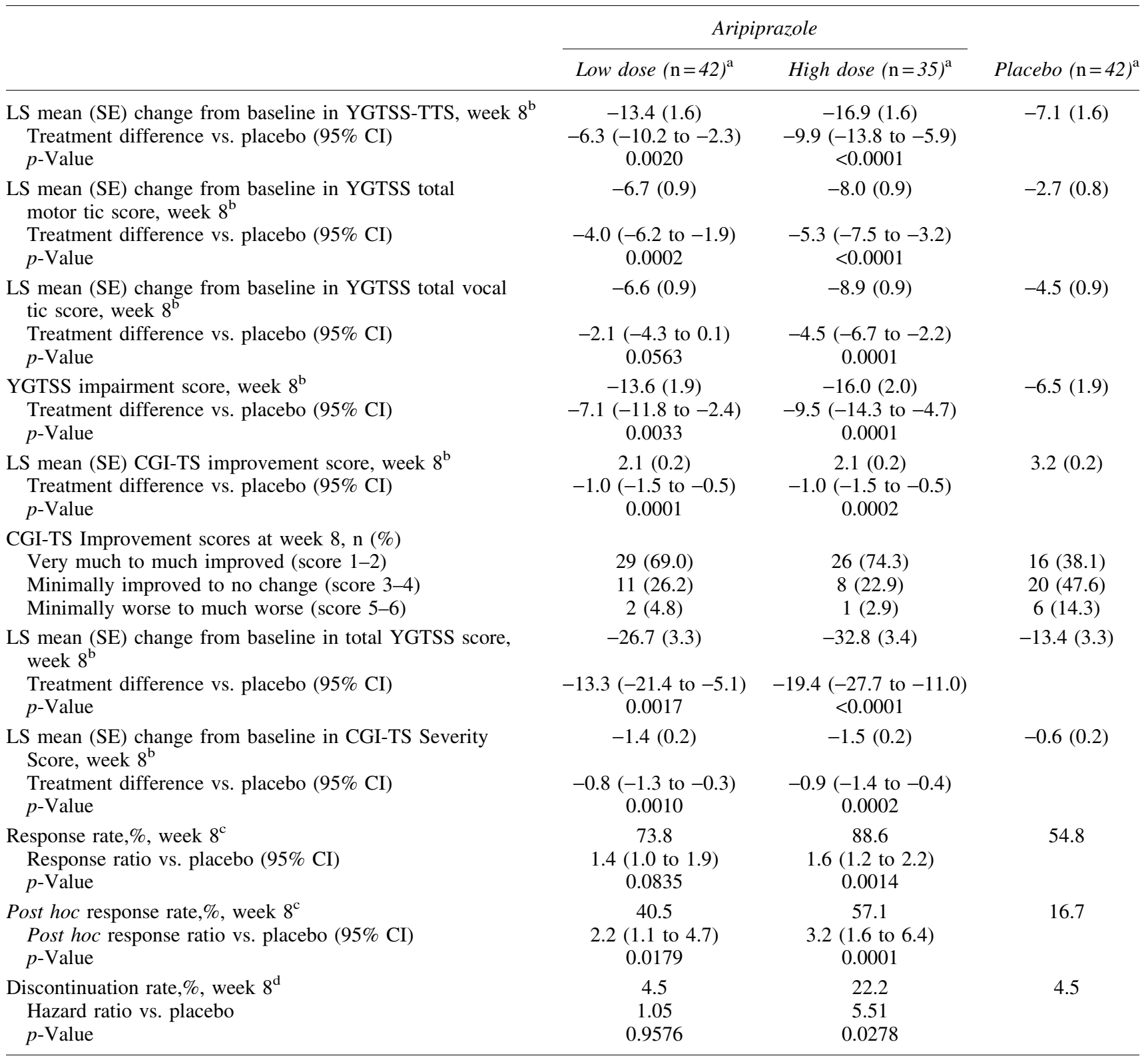

Total YGTSS score ranges from 0 to 100, and CGI-TS score ranges from 1 to 7 . Higher scores represent more severe symptoms, and a greater reduction from baseline represents a greater improvement. Response ratio $>1$ favors aripiprazole. A hazard ratio $<1$ favors aripiprazole.

${ }^{\mathrm{a}}$ Number of patients with a baseline and week 8 assessment used in the mixed model for repeated-measures analysis.

${ }^{\mathrm{b}}$ Derived from a repeated-measures linear model with treatment, week, treatment by week interaction, region, and weight group as fixed categorical effects; the baseline value as a fixed covariate; and the week as the time variable for repeated measures.

${ }^{\mathrm{c}}$ Observed case ITT population. Response rate was defined a priori (>25\% improvement from baseline to endpoint in YGTSS-TTS or a CGI-TS improvement score of one [very much improved] or two [much improved]). A more stringent, post hoc response rate was defined as $>50 \%$ improvement from baseline in YGTSS-TSS.

${ }^{\mathrm{d}}$ Number of patients in the low-dose aripiprazole group, $n=44$, high-dose aripiprazole group, $n=45$, and placebo group, $n=44$. Overall 2 , 10 , and 2 patients discontinued treatment in the low-dose, high-dose, and placebo groups, respectively. $p$-Value derived from Cox proportional hazard regression adjusting for region and weight group.

CGI-TS, Clinical Global Impression-Tourette's Syndrome; ITT, intent-to-treat; LS, least squares; SE, standard error; YGTSS-TTS, Yale Global Tic Severity Scale Total Tic Score.

number needed-to-treat $[\mathrm{NNT}]=5$ ), whereas the response rate in the high-dose aripiprazole group was significantly greater than placebo ( $p=0.0014, \mathrm{NNT}=3$; Table 2 and Supplementary Fig. S6). Using the post hoc definition of response as the percentage of patients with $>50 \%$ improvement from baseline in YGTSS-TTS, a significantly greater percentage of patients treated with low- or high-dose aripiprazole were classified as responders $(40.5 \%$ and
$57.1 \%$, respectively) compared with patients in the placebo group ( $16.7 \%, p=0.0179$ and $p<0.0001$, Table 2).

Two patients each discontinued treatment in the placebo and low-dose groups (hazard ratio for discontinuation vs. placebo: 1.05 , $p=0.9576$ ); 10 patients discontinued treatment in the high-dose group (hazard ratio vs. placebo: 5.51, $p=0.0278$; Fig. 2 and Table 2). In the low-dose group, both patients who discontinued 


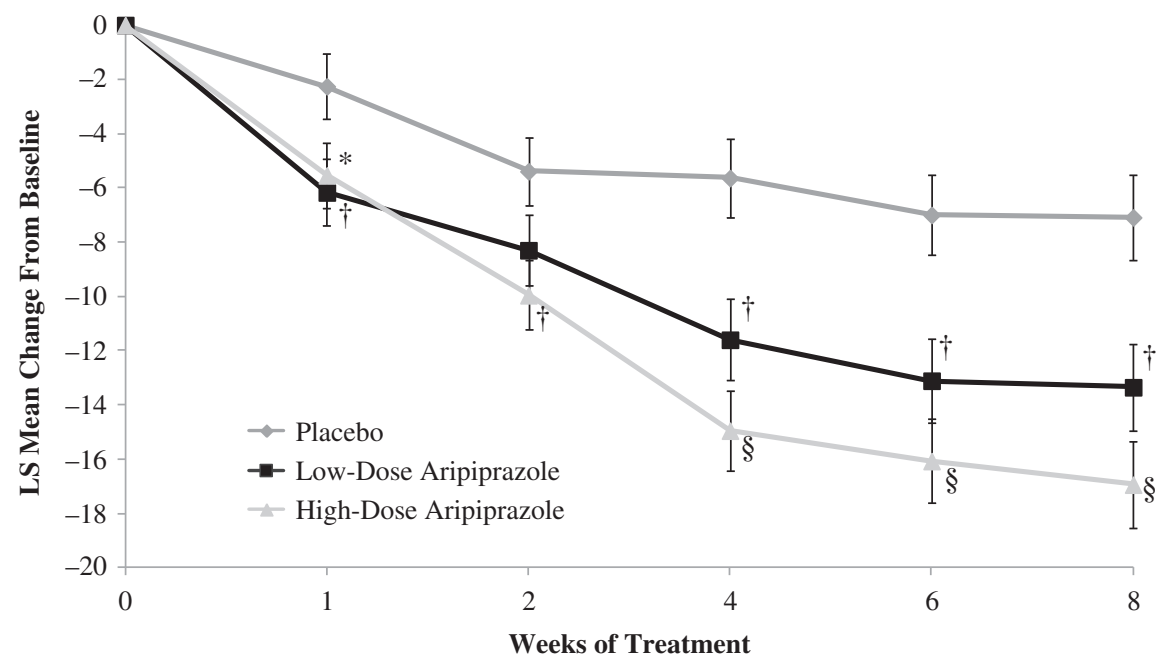

FIG. 3. LS mean (SE) change from baseline in YGTSS-TTS (ITT population). Shown are the LS mean changes from baseline in the YGTSS-TTS by week calculated by using MMRM. Error bars represent the LS mean \pm 1 SE. $* p<0.05 ;{ }^{\dagger} p<0.01 ;{ }^{\S} p<0.0001$ versus placebo. ITT, intent-to-treat; LS, least squares; MMRM, mixed-model repeated measures; SE, standard error; YGTSS-TTS, Yale Global Tic Severity Scale Total Tic Score.

treatment were $<50 \mathrm{~kg}$; in the high-dose group, 9 out of 10 patients were $<50 \mathrm{~kg}$.

High-dose aripiprazole demonstrated statistically significant improvement versus placebo in LS mean change from baseline to week 8 in all SNAP-IV rating subscales (Table 3). No significant difference was noted between low-dose aripiprazole and placebo on any SNAP-IV rating subscale. Neither dose was statistically superior to placebo for the LS mean change from baseline to week 8 in scores on CY-BOCS, CDRS-R, or PARS.

Overall, $65.9 \%, 75.6 \%$, and $40.9 \%$ of patients in the low-dose, high-dose, and placebo groups, respectively, reported $\geq 1 \mathrm{AE}$ (Table 4). Among the aripiprazole treatment groups, the most common AEs were sedation, somnolence, increased appetite, and fatigue. Most events were mild or moderate in severity; no serious AEs or deaths occurred. AEs were the most common reason for discontinuation ( $n=8[6 \%])$; seven out of eight patients who discontinued due to AEs belonged to the high-dose low-weight $(10 \mathrm{mg},<50 \mathrm{~kg})$ group. Fatigue was the only AE to result in discontinuation in $>1$ patient ( $n=2[4.4 \%]$ in the high-dose low-weight group, Table 4).

Extrapyramidal symptom-related AEs (i.e., akathisia, dystonia, extrapyramidal disorder, parkinsonism rest tremor, and tremor) occurred in one (2.3\%), six (13.3\%), and zero patients in the low-dose, high-dose, and placebo groups, respectively. No significant difference in BARS score change from baseline to week 8 was observed between low-dose and high-dose aripiprazole versus placebo (Table 4). A significant improvement was observed in change from baseline to week 8 in SAS total score $(p=0.0357)$ and AIMS score $(p=0.0382)$ for highdose aripiprazole versus placebo; treatment differences between lowdose aripiprazole and placebo were not significant (Table 4).

Per C-SSRS responses, two patients experienced emergence of suicidal ideation (one each in the low-dose and placebo groups), four patients had worsening suicidal ideation (three in the low-dose and one in the placebo groups); no suicidal behavior or ideation with a specific plan was reported. Mean suicidal ideation intensity total scores were low at baseline and demonstrated minimal change at week 8 (Table 3). No suicide-related AEs occurred.

Mean changes from baseline to week 8 in serum chemistry, hematology, and urinalysis laboratory tests were similar across treatment groups. A greater mean [SD] reduction from baseline to week 8 was noted in prolactin levels in the low- and high-dose groups for boys $(-5.82[ \pm 7.25] \mu \mathrm{g} / \mathrm{L}$ and $-4.32[ \pm 6.84] \mu \mathrm{g} / \mathrm{L}$, respectively) and girls $(-15.58[ \pm 23.23] \mu \mathrm{g} / \mathrm{L}$ and $-5.40[ \pm 7.78] \mu \mathrm{g} / \mathrm{L}$, respectively) versus the placebo group (boys, $-1.48[ \pm 7.88] \mu \mathrm{g} / \mathrm{L}$; girls, -0.23 [ \pm 4.66$] \mu \mathrm{g} / \mathrm{L})$. The frequency of potentially clinically relevant laboratory and vital sign abnormalities was similar across groups, except for a higher proportion of patients with potentially clinically relevant weight gain $(\geq 7 \%)$ with low-dose $(18.2 \%)$ than with highdose aripiprazole $(9.3 \%)$ or placebo $(9.1 \%)$. Overall, for the safety sample, the mean change in weight $(\mathrm{kg}, \mathrm{SD})$ from baseline to week 8 was 1.8 (2.0), 1.0 (2.0), and 0.6 (2.1) in the low-dose aripiprazole, high-dose aripiprazole, and placebo groups, respectively.

\section{Discussion}

This study demonstrated the efficacy of high-dose and low-dose aripiprazole for the treatment of tics in children and adolescents with TD. Improvements in the primary (YGTSS-TTS) and key secondary (CGI-TS improvement scale score) endpoints were observed as early as week 1 of treatment and were sustained throughout the study. Overall, a 45.9\% and 54.2\% decrease from baseline in YGTSS-TTS was observed with low- and high-dose aripiprazole, respectively. This magnitude of change ( $>25 \%)$ is predictive of a CGI improvement scale score of 1 or 2 (very much improved or much improved) based on a pooled analysis of two 10-week studies comparing behavioral interventions in patients with TD (mean age, 22.0 years) (Jeon et al. 2013). For all efficacy endpoints, with the exception of the CGI-TS improvement and severity scale scores, the magnitude of improvement was dose related (i.e., greater improvements with high- vs. low-dose aripiprazole).

Analyses using the more stringent post hoc defined response rate were consistent with results from the a priori defined response rate, demonstrating that a greater percentage of patients treated with low- or high-dose oral aripiprazole achieved $>50 \%$ improvement in YGTSS-TTS at week 8 compared with the patients in the placebo group. Of note, when using the more stringent response criteria, the response ratio versus placebo was higher than that using the less stringent a priori defined response rate. The higher response ratio versus placebo using the more stringent criteria reflects the higher placebo response rate using a priori criteria. 
Table 3. Other Outcomes by Treatment Group (Safety Population)

\begin{tabular}{|c|c|c|c|}
\hline & \multicolumn{2}{|c|}{ Aripiprazole } & \multirow[b]{2}{*}{ Placebo $(\mathrm{n}=42)^{\mathrm{a}}$} \\
\hline & Low dose $(\mathrm{n}=42)^{\mathrm{a}}$ & High dose $(\mathrm{n}=35)^{\mathrm{a}}$ & \\
\hline $\begin{array}{l}\text { LS mean (SE) change from baseline in SNAP-IV } \\
\text { inattention average score, week } 8^{\mathrm{b}}\end{array}$ & $-0.40(0.09)$ & $-0.58(0.09)$ & $-0.23(0.09)$ \\
\hline $\begin{array}{l}\text { Treatment difference vs. placebo }(95 \% \mathrm{CI}) \\
p \text {-Value }\end{array}$ & $\begin{array}{c}-0.17(-0.39 \text { to }-0.05) \\
0.1291\end{array}$ & $\begin{array}{c}-0.35(-0.58 \text { to }-0.12) \\
0.0027\end{array}$ & \\
\hline $\begin{array}{l}\text { LS mean (SE) change from baseline in SNAP-IV } \\
\text { hyperactivity/impulsivity average score, week } 8^{\mathrm{b}}\end{array}$ & $-0.38(0.09)$ & $-0.58(0.09)$ & $-0.35(0.09)$ \\
\hline $\begin{array}{l}\text { Treatment difference vs. placebo }(95 \% \text { CI }) \\
p \text {-Value }\end{array}$ & $\begin{array}{c}-0.03(-0.22 \text { to } 0.17) \\
0.8041\end{array}$ & $\begin{array}{c}-0.22(-00.43 \text { to }-0.02) \\
0.0352\end{array}$ & \\
\hline $\begin{array}{l}\text { LS mean (SE) change from baseline in SNAP-IV } \\
\text { ADD/ADHD average score, week } 8^{\mathrm{b}}\end{array}$ & $-0.38(0.08)$ & $-0.57(0.08)$ & $-0.29(0.08)$ \\
\hline $\begin{array}{l}\text { Treatment difference vs. placebo }(95 \% \mathrm{CI}) \\
p \text {-Value }\end{array}$ & $\begin{array}{c}-0.10(-0.29 \text { to } 0.09) \\
0.3148\end{array}$ & $\begin{array}{c}-0.29(-0.48 \text { to }-0.09) \\
0.0048\end{array}$ & \\
\hline $\begin{array}{l}\text { LS mean (SE) change from baseline in CY-BOCS total } \\
\text { score, week } 8^{\mathrm{b}}\end{array}$ & $-0.20(0.56)$ & $-0.74(0.56)$ & $-0.18(0.54)$ \\
\hline $\begin{array}{l}\text { Treatment difference vs. placebo }(95 \% \mathrm{CI}) \\
p \text {-Value }\end{array}$ & $\begin{array}{c}-0.02(-1.28 \text { to } 1.24) \\
0.9785\end{array}$ & $\begin{array}{c}-0.56(-1.84 \text { to } 0.73) \\
0.3937\end{array}$ & \\
\hline $\begin{array}{l}\text { LS mean (SE) change from baseline in CY-BOCS } \\
\text { obsessions total score, week } 8^{\mathrm{b}}\end{array}$ & $0.06(0.31)$ & $-0.27(0.32)$ & $0.14(0.30)$ \\
\hline $\begin{array}{l}\text { Treatment difference vs. placebo }(95 \% \text { CI }) \\
p \text {-Value }\end{array}$ & $\begin{array}{c}-0.07(-0.79 \text { to } 0.65) \\
0.8426\end{array}$ & $\begin{array}{c}-0.41(-1.14 \text { to } 0.33) \\
0.2774\end{array}$ & \\
\hline $\begin{array}{l}\text { LS mean (SE) change from baseline in CY-BOCS } \\
\text { compulsions total score, week } 8^{\mathrm{b}}\end{array}$ & $-0.20(0.31)$ & $-0.29(0.31)$ & $-0.22(0.30)$ \\
\hline $\begin{array}{l}\text { Treatment difference vs. placebo }(95 \% \mathrm{CI}) \\
p \text {-Value }\end{array}$ & $\begin{array}{c}0.02(-0.67 \text { to } 0.70) \\
0.9549\end{array}$ & $\begin{array}{c}-0.07(-0.77 \text { to } 0.64) \\
0.8527\end{array}$ & \\
\hline $\begin{array}{l}\text { LS mean (SE) change from baseline in CDRS-R total score, } \\
\text { week } 8^{\mathrm{b}}\end{array}$ & $-2.58(0.73)$ & $-2.06(0.73)$ & $-1.80(0.72)$ \\
\hline $\begin{array}{l}\text { Treatment difference vs. placebo }(95 \% \text { CI }) \\
p \text {-Value }\end{array}$ & $\begin{array}{c}-0.77(-2.43 \text { to } 0.88) \\
0.3562\end{array}$ & $\begin{array}{c}-0.26(-1.97 \text { to } 1.46) \\
0.7679\end{array}$ & \\
\hline $\begin{array}{l}\text { LS mean (SE) change from baseline in PARS total score, } \\
\text { week } 8^{\mathrm{b}}\end{array}$ & $-0.59(0.62)$ & $-0.88(0.64)$ & $-1.49(0.61)$ \\
\hline Treatment difference vs. placebo $(95 \% \mathrm{CI})$ & $0.90(-0.59$ to 2.39$)$ & $0.62(-0.93$ to 2.16$)$ & \\
\hline$p$-Value & 0.2346 & 0.4306 & \\
\hline
\end{tabular}

SNAP-IV subscale scores range from 0 to 3, CY-BOCS total score ranges from 0 to 40, CY-BOCS obsessions total and compulsions total scores range from 0 to 25 , PARS total score ranges from 0 to 35 , and CDRS-R total score ranges from 17 to 113 . Higher scores represent a worse condition, and a larger reduction from baseline represents greater improvement.

${ }^{a}$ Number of patients with baseline and week 8 assessment used in the mixed model for repeated-measures analysis.

${ }^{\mathrm{b}}$ Derived from a repeated-measures linear model with treatment, week, treatment by week interaction, region, and weight group as fixed categorical effects; the baseline value as a fixed covariate; and the week as the time variable for repeated measures.

ADD/ADHD, attention-deficit disorder/attention-deficit/hyperactivity disorder; CDRS-R, Children's Depression Rating Scale-Revised; C-SSRS, Columbia-Suicide Severity Rating Scale; CY-BOCS, Children's Yale-Brown Obsessive Compulsive Scale; LS, least squares; PARS, Pediatric Anxiety Rating Scale; SE, standard error; SNAP-IV, Swanson, Nolan, and Pelham-IV rating scale.

Improvements at week 8 on the YGTSS-TTS motor tic, vocal tic, and impairment scores were greater in the low- and high-dose aripiprazole groups versus placebo. These findings are consistent with results for the primary endpoint, and they indicate that improvements in all three components contribute to the overall primary outcome. Improvements versus placebo on the primary endpoint were significant in the low-dose and high-dose aripiprazole groups in North America and only in the low-dose aripiprazole group in Europe. The small sample sizes in the rest of the Europe stratum (range for $n$ 's at week 8: 3-5) do not allow for reliable inferences to be made; study sample size estimates did not take into account power calculations for subgroup analyses.

Oral aripiprazole was generally well tolerated. Symptoms associated with psychiatric comorbidities did not worsen with aripiprazole treatment. Although a greater proportion of patients exhibited extrapyramidal symptoms with aripiprazole, only one patient reported parkinsonian resting tremor (in the high-dose group); nevertheless, mean SAS and AIMS scores improved sig- nificantly in the high-dose group versus placebo. The improvement in SAS scores in the aripiprazole groups may partially reflect fewer tics with aripiprazole treatment.

Results from this trial complement other studies of aripiprazole for the treatment of TD. The efficacy of aripiprazole was recently demonstrated in a 10-week study in 61 Korean children with TD, with significant reductions from baseline to study end in YGTSS-TTS and CGI-TS Severity of Illness score versus placebo (Yoo et al. 2013). Further, in a case series of 100 patients with TD (mean age, 27.1 years), aripiprazole treatment considerably reduced tic severity in $82 \%$ of patients, with $99 \%$ of patients reporting positive results (Wenzel et al. 2012). These two studies, along with a third study that assessed aripiprazole's metabolic effect in children (mean age, 11.0 years), reported aripiprazole to be safe for use in children and adolescents with TD (Yoo et al. 2011, 2013; Rizzo et al. 2012; Wenzel et al. 2012).

The AEs observed in this trial are consistent with other pediatric studies of oral aripiprazole in schizophrenia, bipolar I disorder, and 
Table 4. Safety Summary (Safety Population)

\begin{tabular}{|c|c|c|c|}
\hline & \multicolumn{3}{|c|}{ Patients, n (\%) } \\
\hline & \multicolumn{2}{|c|}{ Aripiprazole } & \multirow[b]{2}{*}{ Placebo $(\mathrm{n}=44$} \\
\hline & Low dose $(\mathrm{n}=44)$ & High dose $(\mathrm{n}=45)$ & \\
\hline Any patient with $\geq 1$ adverse event & $29(65.9)$ & $34(75.6)$ & $18(40.9)$ \\
\hline \multicolumn{4}{|l|}{ Most common AEs ( $\geq 5 \%$ in any treatment group) ${ }^{\mathrm{a}}$} \\
\hline Sedation & $8(18.2)$ & $4(8.9)$ & $1(2.3)$ \\
\hline Somnolence & $5(11.4)$ & $7(15.6)$ & $1(2.3)$ \\
\hline Increased appetite & $4(9.1)$ & $3(6.7)$ & $1(2.3)$ \\
\hline Fatigue & $3(6.8)$ & $7(15.6)$ & 0 \\
\hline Headache & $3(6.8)$ & $4(8.9)$ & $1(2.3)$ \\
\hline Nausea & $3(6.8)$ & $4(8.9)$ & $1(2.3)$ \\
\hline Nasopharyngitis & $3(6.8)$ & $4(8.9)$ & 0 \\
\hline Vomiting & $2(4.5)$ & $3(6.7)$ & $2(4.5)$ \\
\hline Lethargy & 0 & 5 (11.1) & 0 \\
\hline Restlessness & 0 & $3(6.7)$ & $1(2.3)$ \\
\hline Akathisia & 0 & $3(6.7)$ & 0 \\
\hline AEs leading to treatment discontinuation ${ }^{\mathrm{ab}}$ & $1(2.3)$ & $7(15.6)$ & $1(2.3)$ \\
\hline Somnambulism & $1(2.3)$ & 0 & 0 \\
\hline Fatigue & 0 & $2(4.4)$ & 0 \\
\hline ECG QT prolonged & 0 & $1(2.2)$ & 0 \\
\hline Disturbance in attention & 0 & $1(2.2)$ & 0 \\
\hline Dysarthria & 0 & $1(2.2)$ & 0 \\
\hline Extrapyramidal disorder & 0 & $1(2.2)$ & 0 \\
\hline Headache & 0 & $1(2.2)$ & 0 \\
\hline Lethargy & 0 & $1(2.2)$ & 0 \\
\hline Somnolence & 0 & $1(2.2)$ & 0 \\
\hline Insomnia & 0 & $1(2.2)$ & 0 \\
\hline TD & 0 & 0 & $1(2.3)$ \\
\hline Extrapyramidal symptom and suicidal ideation assessments & $\mathrm{n}=42$ & $\mathrm{n}=35$ & $\mathrm{n}=42$ \\
\hline LS mean (SE) change from baseline in AIMS score, week $8^{\mathrm{c}}$ & $-3.45(0.87)$ & $-4.26(0.87)$ & $-1.99(0.84)$ \\
\hline Treatment difference vs. placebo $(95 \% \mathrm{CI})$ & $-1.46(-3.57$ to 0.65$)$ & $-2.27(-4.42$ to -0.13$)$ & \\
\hline$p$-Value & 0.1717 & 0.0382 & \\
\hline LS mean (SE) change from baseline in BARS total score, week $8^{c}$ & $-0.18(0.05)$ & $-0.10(0.05)$ & $-0.09(0.05)$ \\
\hline Treatment difference vs. placebo $(95 \% \mathrm{CI})$ & $-0.09(-0.20$ to 0.03$)$ & $-0.01(-0.13$ to 0.12$)$ & \\
\hline$p$-Value & 0.1483 & 0.9090 & \\
\hline LS mean (SE) change from baseline in SAS total score, week $8^{\mathrm{c}}$ & $-0.28(0.22)$ & $-0.74(0.22)$ & $-0.10(0.21)$ \\
\hline Treatment difference vs. placebo $(95 \% \mathrm{CI})$ & $-0.18(-0.77$ to 0.41$)$ & $-0.64(-1.24$ to -0.04$)$ & \\
\hline$p$-Value & 0.5502 & 0.0357 & \\
\hline Mean (SD) change from baseline in C-SSRS score, week $8^{\mathrm{d}}$ & $-0.3(1.5)$ & $0.1(0.8)$ & $0(0)$ \\
\hline
\end{tabular}

AIMS scores range from 0 to 28 , BARS total score ranges from 0 to 5 , and SAS total score ranges from 0 to 40 . Higher scores represent a worse condition, and a larger reduction from baseline represents greater improvement.

Suicidal ideation intensity total score is the sum of intensity scores ranging from 1 (least severe) to 5 (most severe) of five items (frequency, duration, controllability, deterrents, and reasons for ideation). A score of 0 is given if no suicidal ideation is reported for a total score ranging from 0 to 25 .

${ }^{a}$ By MedDRA Preferred term. Patients were counted once, per preferred term, for the most severe of multiple occurrences of a specific adverse event. Any patients with AEs in multiple system organ classes were counted once toward the total.

${ }^{\mathrm{b}}$ The patient in the low-dose aripiprazole group and six out of seven patients in the high-dose aripiprazole group who discontinued study medication because of AEs weighed $<50 \mathrm{~kg}$.

${ }^{c}$ Number of patients with baseline and week 8 assessment (ITT population). Derived from a repeated-measures linear model with treatment, week, treatment by week interaction, region, and weight group as fixed categorical effects; the baseline value as a fixed covariate; and the week as the time variable for repeated measures.

${ }^{\mathrm{d}}$ Using suicidal ideation intensity total score.

AEs, adverse events; AIMS, Abnormal Involuntary Movement Scale; BARS, Barnes Akathisia Rating Scale; C-SSRS, Columbia-Suicide Severity Rating Scale; ECG, electrocardiogram; ITT, intent-to-treat; LS, least squares; SAS, Simpson-Angus Scale; SD, standard deviation; SE, standard error; TD, Tourette's disorder.

autistic disorder (Findling et al. 2008, 2009, 2013; Marcus et al. 2009; Owen et al. 2009). Although a higher rate of discontinuations was observed with high-dose aripiprazole versus low-dose aripiprazole or placebo, this rate was consistent with short-term (i.e., 4-8 weeks) pediatric studies of oral aripiprazole (mean ages, 9.3-
15.4 years) at doses of 5-, 10-, 15-, or $30 \mathrm{mg} /$ day (discontinuation rates, 13.0\%-22.2\%) (Findling et al. 2008, 2009; Marcus et al. 2009; Owen et al. 2009) and a 4-week risperidone trial in pediatric and adult patients (mean age, 24.7 years) with TD (discontinuation rate, 21.0\%) (Bruun and Budman 1996). Overall, the AE profile 
reported in this trial demonstrates that oral aripiprazole is safe for use in children and adolescents with TD and may potentially be better tolerated than other approved treatments for tic disorders (e.g., haloperidol) (Shapiro et al. 1973; Sallee et al. 1997).

Other atypical antipsychotics, including quetiapine (Copur et al. 2007; Huys et al. 2012) and risperidone (Huys et al. 2012), have consistently reported efficacy for the treatment of tics. Quetiapine treatment resulted in a $65 \%$ and $74 \%$ reduction in the tic severity at weeks 4 and 8, respectively (Copur et al. 2007). Patients treated for 8 weeks with risperidone showed a $32 \%$ reduction in tic severity from baseline compared with a 7\% reduction with placebo (Scahill et al. 2003). However, safety concerns remain, including weight gain, fatigue, and overall tolerability with these drugs. Different antipsychotics differ in their potential to induce side effects such as weight gain and cardiovascular risks; aripiprazole has a low potential to induce metabolic side effects, but as with other second-generation antipsychotics, regular monitoring is warranted (De Hert et al. 2011, 2012).

Guidelines for the treatment of tic disorders recommend a hierarchical, multimodal approach (Roessner et al. 2011; Pringsheim et al. 2012; Murphy et al. 2013). Patients should be carefully evaluated for coexisting conditions, and initial treatment selection should prioritize the condition causing the most impairment, which in some cases may be a comorbid psychiatric disorder (Roessner et al. 2011; Murphy et al. 2013). Treatment guidelines recommend consideration of both behavioral and pharmacologic approaches (Roessner et al. 2011; Verdellen et al. 2011; Pringsheim et al. 2012; Steeves et al. 2012; Murphy et al. 2013). The randomized controlled Comprehensive Behavioral Intervention for Tics (C-BIT) study, which evaluated a behavioral intervention based on habit reversal, demonstrated a 7.6point reduction in YGTSS-TTS after 10 weeks of treatment, a significant improvement over the 3.5-point reduction attained with the control treatment ( $p<0.001$; effect size, 0.68$)$ (Piacentini et al. 2010).

There are no studies directly comparing C-BIT or other forms of habit reversal therapy with pharmacologic or combined therapies in youth with TD. However, based on its demonstrated effectiveness and low risk of adverse effects, habit reversal is recommended as a first-line treatment option (Pringsheim et al. 2012; Steeves et al. 2012). Comparative studies of behavioral and pharmacologic approaches could advance the therapeutics of TD and extend the reach of treatment guidelines. Currently, however, a careful appraisal of risk-benefit profiles as well as preference and availability can guide physicians in selecting the most appropriate treatment modality for their patient (Pringsheim et al. 2012; Steeves et al. 2012; Murphy et al. 2013).

This study was one of the studies that led to the approval of aripiprazole by the FDA for the treatment of TD. Dosing adjustments were recommended in the US Prescribing Information based on the results from this study. For patients weighing $<50 \mathrm{~kg}$, the recommended initiation dose is $2 \mathrm{mg} /$ day, with a target of $5 \mathrm{mg} /$ day and a maximum dose of $10 \mathrm{mg} /$ day. For patients weighing $\geq 50 \mathrm{~kg}$, it is recommended to initiate dosing at $2 \mathrm{mg} /$ day and increase it to $10 \mathrm{mg} /$ day on day 8 , with the maximum dose being $20 \mathrm{mg} /$ day (Abilify ${ }^{\circledR} 2016$ ).

To date, this study is the largest double-blind, placebocontrolled, multinational trial in children and adolescents with TD. Relative to some phase 3 trials in other disease states, this sample size was small, and this study was not powered to detect statistically significant treatment differences within subgroups. However, it is difficult to recruit children and adolescents for clinical trials, and the potential study population is much smaller for rare diseases. Future research studies should consider primary analyses of patient subgroups (e.g., comorbid conditions) or pooling data across trials to obtain greater power to detect treatment differences.

\section{Conclusions}

Our findings support the efficacy and safety of aripiprazole for children and adolescents with TD. With the FDA approval in December 2014, aripiprazole represents a validated option for treating tics in children and adolescents with TD.

\section{Clinical Significance}

This was a randomized, double-blind, placebo-controlled study that demonstrated the efficacy and safety of high-dose and lowdose aripiprazole for the treatment of symptoms in children and adolescents with TD. Overall, a $45.9 \%$ and $54.2 \%$ decrease from baseline in total tic score was observed with low- and high-dose aripiprazole, respectively. A consistent and significantly greater response rate versus placebo was observed in both aripiprazole dose groups by using both standard and more stringent definitions of response. These results are consistent with findings from other smaller studies and studies performed in other regions of the world (e.g., Asia) in children and adolescents with TD. The U.S. FDA approval of oral aripiprazole in December 2014 provided a new option for the first time in $>30$ years for the treatment of tics in children and adolescents with TD. Oral aripiprazole might be considered a suitable treatment option for patients and caregivers with tolerability concerns.

\section{Acknowledgments}

The initial draft of this article was written by Jessica Holzhauer, DVM, with assistance from Amy Roth Shaberman, $\mathrm{PhD}$, medical writers from C4 MedSolutions, LLC (Yardley, PA), a CHC Group company, with funding from Otsuka Pharmaceutical Development \& Commercialization, Inc.

\section{Authors' Contributions}

The corresponding author had full access to all the data in the study and had final responsibility for the decision to submit it for publication. All authors contributed to the preparation of this article. Floyd Sallee contributed to interpretation of the data. Eva Kohegyi, Robert McQuade, Raymond Sanchez, William Carson, and Roger Kurlan (principal investigator) designed the study and oversaw the execution and completion of the study. Joan Zhao performed the statistical analyses. Kevin Cox, Alet van Beek, and Margaretta Nyilas oversaw the execution and completion of the study.

\section{Disclosures}

Floyd Sallee reports personal fees from Otsuka Pharmaceutical Development \& Commercialization, Inc. and Astra-Zeneca Pharmaceutical, and serves on the board of directors for P2D Bioscience. Eva Kohegyi, Joan Zhao, Robert McQuade, Kevin Cox, Raymond Sanchez, Margaretta Nyilas, and William Carson are employees of Otsuka Pharmaceutical Development \& Commercialization, Inc. Alet van Beek was employed by Otsuka Pharmaceutical Development \& Commercialization, Inc. at the time that this research was conducted. Roger Kurlan has nothing to disclose.

\section{References}

Abilify $^{\circledR}$ (aripiprazole): Full Prescribing Information. Tokyo, Japan, Otsuka Pharmaceutical Co., Ltd., 2016.

American Psychiatric Association: Diagnostic and Statistical Manual of Mental Disorders, 4th ed. Washington, DC: American Psychiatric Association; 1994. 
American Psychiatric Association: Diagnostic and Statistical Manual of Mental Disorders, 4th ed., Text Revision. Washington, DC: American Psychiatric Association; 2000.

Bitsko RH, Holbrook JR, Visser SN, Mink JW, Zinner SH, Ghandour RM, Blumberg SJ: A national profile of Tourette syndrome, 20112012. J Dev Behav Pediatr 35:317-322, 2014.

Bruun RD, Budman CL: Risperidone as a treatment for Tourette's syndrome. J Clin Psychiatry 57:29-31, 1996.

Copur M, Arpaci B, Demir T, Narin H: Clinical effectiveness of quetiapine in children and adolescents with Tourette's syndrome: A retrospective case-note survey. Clin Drug Investig 27:123-130, 2007.

Cutler D, Murphy T, Gilmour J, Heyman I: The quality of life of young people with Tourette syndrome. Child Care Health Dev 35:496-504, 2009.

De Hert M, Detraux J, van Winkel R, Yu W, Correll CU: Metabolic and cardiovascular adverse effects associated with antipsychotic drugs. Nat Rev Endocrinol 8:114-126, 2012.

De Hert M, Dobbelaere M, Sheridan EM, Cohen D, Correll CU: Metabolic and endocrine adverse effects of second-generation antipsychotics in children and adolescents: A systematic review of randomized, placebo controlled trials and guidelines for clinical practice. Eur Psychiatry 26:144-158, 2011.

Dutta N, Cavanna AE: The effectiveness of habit reversal therapy in the treatment of Tourette syndrome and other chronic tic disorders: A systematic review. Funct Neurol 28:7-12, 2013.

Findling RL, Correll CU, Nyilas M, Forbes RA, McQuade RD, Jin N, Ivanova S, Mankoski R, Carson WH, Carlson GA: Aripiprazole for the treatment of pediatric bipolar I disorder: A 30-week, randomized, placebo-controlled study. Bipolar Disord 15:138-149, 2013.

Findling RL, Nyilas M, Forbes RA, McQuade RD, Jin N, Iwamoto T, Ivanova S, Carson WH, Chang K: Acute treatment of pediatric bipolar I disorder, manic or mixed episode, with aripiprazole: A randomized, double-blind, placebo-controlled study. J Clin Psychiatry 70:1441-1451, 2009.

Findling RL, Robb A, Nyilas M, Forbes RA, Jin N, Ivanova S, Marcus R, McQuade RD, Iwamoto T, Carson WH: A multiple-center, randomized, double-blind, placebo-controlled study of oral aripiprazole for treatment of adolescents with schizophrenia. Am J Psychiatry 165:1432-1441, 2008.

Haloperidol Tablet: Full Prescribing Information. Morgantown, WV, Mylan Pharmaceuticals, Inc., 2011.

Huys D, Hardenacke K, Poppe P, Bartsch C, Baskin B, Kuhn J: Update on the role of antipsychotics in the treatment of Tourette syndrome. Neuropsychiatr Dis Treat 8:95-104, 2012.

Jeon S, Walkup JT, Woods DW, Peterson A, Piacentini J, Wilhelm S, Katsovich L, McGuire JF, Dziura J, Scahill L: Detecting a clinically meaningful change in tic severity in Tourette syndrome: A comparison of three methods. Contemp Clin Trials 36:414-420, 2013.

Leckman JF, Riddle MA, Hardin MT, Ort SI, Swartz KL, Stevenson J, Cohen DJ: The Yale Global Tic Severity Scale: Initial testing of a clinician-rated scale of tic severity. J Am Acad Child Adolesc Psychiatry 28:566-573, 1989.

Leckman JF, Zhang H, Vitale A, Lahnin F, Lynch K, Bondi C, Kim YS, Peterson BS: Course of tic severity in Tourette syndrome: The first two decades. Pediatrics 102:14-19, 1998.

Marcus RN, Owen R, Kamen L, Manos G, McQuade RD, Carson WH, Aman MG: A placebo-controlled, fixed-dose study of aripiprazole in children and adolescents with irritability associated with autistic disorder. J Am Acad Child Adolesc Psychiatry 48:1110-1119, 2009.

Murphy TK, Lewin AB, Storch EA, Stock S: Practice parameter for the assessment and treatment of children and adolescents with tic disorders. J Am Acad Child Adolesc Psychiatry 52:1341-1359, 2013.
Owen R, Sikich L, Marcus RN, Corey-Lisle P, Manos G, McQuade RD, Carson WH, Findling RL: Aripiprazole in the treatment of irritability in children and adolescents with autistic disorder. Pediatrics 124:1533-1540, 2009.

Piacentini J, Woods DW, Scahill L, Wilhelm S, Peterson AL, Chang S, Ginsburg GS, Deckersbach T, Dziura J, Levi-Pearl S, Walkup JT: Behavior therapy for children with Tourette disorder: A randomized controlled trial. JAMA 303:1929-1937, 2010.

Pringsheim T, Doja A, Gorman D, McKinlay D, Day L, Billinghurst L, Carroll A, Dion Y, Luscombe S, Steeves T, Sandor P: Canadian guidelines for the evidence-based treatment of tic disorders: Pharmacotherapy. Can J Psychiatry 57:133-143, 2012.

Rizzo R, Eddy CM, Cali P, Gulisano M, Cavanna AE: Metabolic effects of aripiprazole and pimozide in children with Tourette syndrome. Pediatr Neurol 47:419-422, 2012.

Roessner V, Plessen KJ, Rothenberger A, Ludolph AG, Rizzo R, Skov L, Strand G, Stern JS, Termine C, Hoekstra PJ: European clinical guidelines for Tourette syndrome and other tic disorders. Part II: Pharmacological treatment. Eur Child Adolesc Psychiatry 20:173-196, 2011.

Sallee FR, Nesbitt L, Jackson C, Sine L, Sethuraman G: Relative efficacy of haloperidol and pimozide in children and adolescents with Tourette's disorder. Am J Psychiatry 154:1057-1062, 1997.

Scahill L, Leckman JF, Schultz RT, Katsovich L, Peterson BS: A placebo-controlled trial of risperidone in Tourette syndrome. Neurology 60:1130-1135, 2003.

Shapiro AK, Shapiro E, Wayne H: Treatment of Tourette's syndrome with haloperidol, review of 34 cases. Arch Gen Psychiatry 28:92-97, 1973.

Steeves T, McKinlay BD, Gorman D, Billinghurst L, Day L, Carroll A, Dion Y, Doja A, Luscombe S, Sandor P, Pringsheim T: Canadian guidelines for the evidence-based treatment of tic disorders: Behavioural therapy, deep brain stimulation, and transcranial magnetic stimulation. Can J Psychiatry 57:144-151, 2012.

Steeves TD, Fox SH: Neurobiological basis of serotonin-dopamine antagonists in the treatment of Gilles de la Tourette syndrome. Prog Brain Res 172:495-513, 2008.

US Food and Drug Administration: Orphan Drug Act: Available at: www.fda.gov/RegulatoryInformation/Legislation/Significant AmendmentstotheFDCAct/OrphanDrugAct/default.htm (last accessed August 24, 2015), 2013.

US Food and Drug Administration: Pimozide approved for Tourette's syndrome: FDA Drug Bull 14:24-25, 1984.

Verdellen C, van de Griendt J, Hartmann A, Murphy T: European clinical guidelines for Tourette syndrome and other tic disorders. Part III: Behavioural and psychosocial interventions. Eur Child Adolesc Psychiatry 20:197-207, 2011.

Wenzel C, Kleimann A, Bokemeyer S, Muller-Vahl KR: Aripiprazole for the treatment of Tourette syndrome: A case series of $100 \mathrm{pa}-$ tients. J Clin Psychopharmacol 32:548-550, 2012.

Yoo HK, Joung YS, Lee JS, Song DH, Lee YS, Kim JW, Kim BN, Cho SC: A multicenter, randomized, double-blind, placebocontrolled study of aripiprazole in children and adolescents with Tourette's disorder. J Clin Psychiatry 74:e772-e780, 2013.

Yoo HK, Lee JS, Paik KW, Choi SH, Yoon SJ, Kim JE, Hong JP: Open-label study comparing the efficacy and tolerability of aripiprazole and haloperidol in the treatment of pediatric tic disorders. Eur Child Adolesc Psychiatry 20:127-135, 2011.

Address correspondence to:

Floyd Sallee, MD

Department of Psychiatry

University of Cincinnati College of Medicine

260 Stetson St., Suite 3200

Cincinnati, OH 45219

E-mail: floyd.sallee@uc.edu 